\title{
The Predictive Value of Tp-ec, Q-Tc, Tp-e/Q-T and HRV in Malignant Ventricular Arrhythmia
}

\author{
Guijun Xie, Songwen Zhang* \\ Department of Electrocardiography, The Hefei Affiliated Hospital of Anhui Medical University, Hefei, China \\ Email: ^2198817030@qq.com
}

How to cite this paper: Xie G.J. and Zhang, S.W. (2021) The Predictive Value of Tp-ec, Q-Tc, Tp-e/Q-T and HRV in Malignant Ventricular Arrhythmia. Journal of Biosciences and Medicines, 9, 22-28. https://doi.org/10.4236/jbm.2021.97004

Received: June 2, 2021

Accepted: July 9, 2021

Published: July 12, 2021

Copyright $\odot 2021$ by author(s) and Scientific Research Publishing Inc. This work is licensed under the Creative Commons Attribution International License (CC BY 4.0).

http://creativecommons.org/licenses/by/4.0/

\begin{abstract}
Objective: To explore the predictive ability of Tp-ec, Q-Tc, Tp-e/Q-T and HRV on malignant arrhythmia during hospitalization. Method: 100 patients with malignant ventricular arrhythmia were included as the experimental group, another 100 patients without malignant ventricular arrhythmia were included as control group. The differences of Tp-ec, Q-Tc, Tp-e/Q-T and HRV were compared between the two groups. Multivariate logistic regression analysis was used to study variables and establish prediction model. ROC curve was used to evaluate the predictive ability and best predictive value of each index for malignant ventricular arrhythmia in hospital. Result: Compared with the control group, Tp-ec, Q-Tc, Tp-e/Q-T and HRV in the experimental group were significantly increased, $(\mathrm{P}<0.001), \mathrm{HRV}$ was decreased significantly. Multivariate logistic regression showed that the increase of Tp-ec, Q-Tc, Tp-e/Q-T and the decrease of HRV were the risk factors of malignant ventricular ventricular arrhythmia in hospital $(\mathrm{OR}=11.169,1.788$, $1.001,0.780)$, and bulid prediction model $\mathrm{Z}=-254.827+0.203 * \mathrm{Tp}-\mathrm{ec}+$ $0.581{ }^{*} \mathrm{Q}-\mathrm{Tc}+878.066 * \mathrm{Tp}-\mathrm{e} / \mathrm{Q}-\mathrm{T}-0.248 * \mathrm{SDNN}$. ROC curve showed that the area under the curve (AUC) of TP EC, Q-Tc, Tp-e/Q-T, HRV and predictive model for the diagnosis of malignant ventricular ventricular arrhythmia in hospital were $0.988,0.905,0.973,0.901,0.993$, the best critical values were $100.365 \mathrm{~ms}, 447.078 \mathrm{~ms}, 0.239,100.500,181.792$. Conclusion: The decrease of Tp-ec, Q-Tc, Tp-e/Q-T and HRV were the risk factors of malignant ventricular arrhythmia, and has predictive value for malignant ventricular arrhythmia in hospital. The prediction model combined with Tp-ec, Q-Tc, Tp-e/Q-T and HRV can improve the prediction ability of variables on malignant ventricular arrhythmia in hospital.
\end{abstract}

\section{Keywords}

Tp-ec, Q-Tc, Tp-e/Q-T, HRV, Malignant Ventricular Arrhythmia 


\section{Introduction}

Sudden cardiac death (SCD) is the consequence of malignant progression of various heart diseases, including coronary heart disease, cardiomyopathy, Brugada syndrome, long QT syndrome, etc. Epidemiological studies showed that SCD accounted for $75 \%$ of all kinds of sudden death, malignant ventricular arrhythmia is an important mechanism of SCD. Tp-ec is the time intervals between the top of $\mathrm{T}$ wave and the end of $\mathrm{T}$ wave in ECG after heart rate correction [1], its formation mechanism is that endocardial cells, middle-layer cells ( $\mathrm{M}$ cells) and epicardial cells of ventricular myocytes have different electrophysiological characteristics. There was potential difference in the two-phase plateau phase of action potential in three-layer cardiomyocytes. The potential difference between $\mathrm{M}$ cells and epicardial cells forms the ascending, apex and descending branches of $\mathrm{T}$ wave in surface electrocardiogram with the participation of endocardial potential difference. The $\mathrm{T}$-wave reaches its peak when epicardial repolarization ended, the $\mathrm{T}$ wave decreased to the equipotential line when $\mathrm{M}$ cell repolarization ended. This action potential duration difference, that is, the difference between the repolarization speed of ventricular myocytes, is called ventricular transmural repolarization dispersion (TDR) [2]. Relevant studies show that TDR is closely related to malignant ventricular arrhythmia [3]. Tp-ec, Tp-e/Q-T can indirectly reflect TDR. Q-Tc is based on the corrected Q-T interval of heart rate, excluding the influence of heart rate on Q-T interval. It reflects the general process of depolarization and repolarization of ventricular myocytes and corresponds to the total refractory period of ventricular myocytes. Studies have shown that prolongation of QTd is related to the severity of myocardial fibrosis after myocardial infarction [4]. Heart rate variability (HRV) refers to the changes of ECG R-R interval based on fast Fourier transform. It can reflect the activity of autonomic nerve and balance between cardiac vagus and sympathetic nerves. HRV can be used to predict the progress and prognosis of heart disease, epilepsy, diabetes and other diseases [5]. The purpose of this study is to analyze the relationship between Tp-ec, Q-Tc, Tp-e/Q-T, HRV and malignant ventricular arrhythmia, establish the prediction model by synthesizing all indexes, and evaluate the comprehensive predictive value of each index with the use of receiver operating characteristic curve (ROC curve).

\section{Materials and Methods}

100 patients (48 males and 52 females) who had malignant ventricular arrhythmia and 100 patients (56 males and 46 females) who had no malignant ventricular arrhythmia were collected from January 2000 to may 2021 in the second people's Hospital of Hefei. Inclusion criteria: 1) Inpatients aged 18 years or older and who underwent DCG during hospital; 2) Patients with ventricular fibrillation, ventricular tachycardia, pleomorphic ventricular premature beat and RonT ventricular premature beat (malignant ventricular arrhythmia group). Exclusion criteria: 1) Patients who have taken metoprolol, bisoprolol, 
amiodarone and other drugs which may affect the Tp-e interval and Q-T interval in the past 1 month; 2) Patients with chronic atrial fibrillation and atrial flutter; 3) Patients with ectopic heart beats $>300$ beats per day, significant sinus bradycardia, sinus atrial block of degree II or above, atrioventricular block (Healthy control group).

Analyzing the admission ECG, ECG in-hospital and Holter retrospectively with the use of Shenzhen Boying ECG and Holter system. Ventricular rhythm, Q-T interval and Q-Tc interval were collected according to the ECG of the patients, and Tp-e interval of precordial lead was measured (The intverval from the top of $\mathrm{T}$ wave to the end of $\mathrm{T}$ wave), and taking the average. The $\mathrm{Tp}$-ec and Tp-e/Q-T interval was calculated according to the formula. SDNN was collected according to dynamic electrocardiogram during hospitalization. The Tp-ec, Q-Tc, Tp-e/Q-T, SDNN of the total sample were Compared. Multivariate logistic regression analysis was used to analyze the correlation between Tp-ec, Q-Tc, Tp-e/Q-T, SDNN and the occurrence of malignant ventricular arrhythmia in hospital, and get the prediction model. ROC curve was used to detect Tp-ec, Q-Tc, Tp-e/Q-T, SDNN and predictive model in the diagnosis of malignant ventricular arrhythmia.

\section{Statistical Methods}

Spss25.0 software was used for data processing and statistical analysis. The measurement data of the whole sample are based on the average \pm Standard deviation. T test was used to compare the indexes between groups. Multivariate logistic regression analysis was used to obtain the predictive model of Tp-ec, Q-Tc, Tp-e/Q-T and SDNN for malignant ventricular arrhythmia, and draw the ROC curves of TP EC, Q-Tc, Tp-e/Q-T, SDNN and predictive model. The difference was statistically significant $(\mathrm{P}<0.05)$.

\section{Results}

\subsection{Comparison of Each Index between Groups}

There were differences in Tp-ec, Q-Tc, Tp-e/Q-T and SDNN between malignant ventricular arrhythmia group and healthy control group $(\mathrm{p}<0.001)$. Compared with the control group, Tp-ec, Q-Tc, tp-e/q-t in the malignant ventricular arrhythmia group were larger than those in the healthy control group, and SDNN was smaller. As shown in Table 1.

\subsection{Regression Relationship between Tp-ec, Q-Tc, Tp-e/Q-T, SDNN and Malignant Ventricular Arrhythmia in Hospital}

It suggests that there is regression relationship between $\mathrm{Tp}-\mathrm{ec}, \mathrm{Q}-\mathrm{Tc}, \mathrm{Tp}-\mathrm{e} / \mathrm{Q}-\mathrm{T}$, SDNN and the occurrence of malignant ventricular arrhythmia. The regression relationship was Tp-ec, Q-Tc, Tp-e/Q-T, SDNN in proper sequence. The prediction model is $\mathrm{P}=1 /\left(1+\mathrm{e}^{-\mathrm{z}}\right), \mathrm{Z}=-254.827+0.203 * \mathrm{Tp}-\mathrm{ec}+0.581 * \mathrm{Q}-\mathrm{Tc}+$ $878.066{ }^{*}$ Tp-e/Q-T-0.248* SDNN. As shown in Table 2. 
Table 1. Comparison of each index between the two groups.

\begin{tabular}{ccccccc}
\hline & \multicolumn{2}{c}{ Healthy control group } & \multicolumn{2}{c}{$\begin{array}{c}\text { Malignant ventricular } \\
\text { arrhythmia group }\end{array}$} & t & P value \\
\cline { 2 - 5 } & Mean & standard deviation & Mean & standard deviation & & \\
\hline Tp-ec(mm) & 75.97 & 16.67 & 141.59 & 45.10 & -14.07 & $<0.001$ \\
Q-Tc $(\mathrm{mm})$ & 421.50 & 20.48 & 484.95 & 107.34 & -5.81 & $<0.001$ \\
$\mathrm{Tp}-\mathrm{e} / \mathrm{Q}-\mathrm{T}$ & 0.18 & 0.04 & 0.29 & 0.05 & -17.88 & $<0.001$ \\
$\mathrm{SDNN}$ & 127.40 & 35.18 & 81.50 & 12.39 & 12.307 & $<0.001$ \\
\hline
\end{tabular}

Table 2. Multivariate logistic regression analysis of indexes and the occurrence of malignant ventricular arrhythmia.

\begin{tabular}{ccccccc}
\hline & $\mathrm{B}$ & $\mathrm{S}_{\mathrm{E}}$ & Wald & $\mathrm{P}$ & OR & $95 \% \mathrm{CI}$ \\
\hline Tp-ec & 0.203 & 0.777 & 5.238 & 0.022 & 11.169 & $0.037-0.775$ \\
Q-Tc & 0.581 & 0.252 & 5.330 & 0.021 & 1.788 & $1.092-2.929$ \\
Tp-e/Q-T & 878.066 & 366.734 & 5.733 & 0.017 & 1.001 & $1.494 \mathrm{E}+69-0.000$ \\
SDNN & -0.248 & 0.101 & 6.003 & 0.014 & 0.780 & $0.640-0.952$ \\
prediction model & -254.827 & 108.240 & 5.543 & 0.019 & & \\
\hline
\end{tabular}

\subsection{Diagnostic Value of Tp-ec, Q-Tc, Tp-e/Q-T, SDNN and Predictive Model in the Occurrence of Malignant Ventricular Arrhythmia in Hospital}

ROC curve showed that AUC of Tp-ec, Q-Tc, Tp-e/Q-T, SDNN and predictive model were $0.988,0.905,0.973,0.901,0.993$ respectively. According to the Youden index (Sen + Spe-1), the optimal critical values are $100.365 \mathrm{~mm}, 447.078$ $\mathrm{mm}, 0.239,100.500$ and 181.792, respectively. From high to low, the predictive value of the diagnosis of malignant ventricular arrhythmia was predictive model, Tp-ec, Tp-e/Q-T, Q-Tc, SDNN (see Table 3 and Table 4).

\section{Discussion}

Ventricular muscle can be divided into epicardial cells, endocardial cells and M cells between them, $M$ cells had larger late inward potassium currents and smaller slowly activated delayed rectifier potassium currents. At the early stage of repolarization, the action potential showed a typical peak dome shape. There were differences in electrophysiology among the three layers of ventricular myocytes, which lead to the different duration of action potential (APD). The APD of epicardial cells was the shortest, M cells was the longest, and endocardial cells' were between them, that is, epicardial cells repolarized the earliest and $\mathrm{M}$ cells repolarized the latest [6]. The difference of APD among epicardial cells, endocardial cells and $\mathrm{M}$ cells leads to the inconsistency of transmural repolarization, which is called transmural repolarization dispersion (TDR). Studies have shown that the increase of TDR is one of the mechanisms of ventricular arrhythmia [7]. 
Table 3. AUC of each index in malignant arrhythmia.

\begin{tabular}{ccccc}
\hline & AUC & Se & P & $95 \%$ CI \\
\hline Tp-ec & 0.988 & 0.006 & $<0.001$ & $0.977-0.998$ \\
Q-Tc & 0.905 & 0.021 & $<0.001$ & $0.863-0.947$ \\
Tp-e/Q-T & 0.973 & 0.009 & $<0.001$ & $0.954-0.991$ \\
SDNN & 0.901 & 0.024 & $<0.001$ & $0.854-0.947$ \\
$\begin{array}{c}\text { prediction } \\
\text { model }\end{array}$ & 0.993 & 0.004 & $<0.001$ & $0.985-1.000$ \\
\hline
\end{tabular}

Table 4. Predictive cut-off value of each index for malignant ventricular arrhythmia.

\begin{tabular}{ccccccc}
\hline & Cut-off & TPR & TNR & YI & PPV & NPV \\
\hline Tp-ec & 100.365 & 0.960 & 0.940 & 0.900 & $92 \%$ & $92 \%$ \\
Q-Tc & 447.078 & 0.770 & 0.900 & 0.670 & $88.51 \%$ & $79.65 \%$ \\
Tp-e/Q-T & 0.239 & 0.890 & 0.960 & 0.850 & $91.76 \%$ & $80.87 \%$ \\
SDNN & 100.500 & 0.780 & 0.980 & 0.770 & $81.82 \%$ & $98.73 \%$ \\
prediction model & 181.792 & 0.990 & 0.940 & 0.930 & $91.42 \%$ & $95.79 \%$ \\
\hline
\end{tabular}

Relevant studies have shown that the peak of $\mathrm{T}$ wave in surface ECG is consistent with the end of epicardial cell repolarization, and the end of $\mathrm{T}$ wave is consistent with the end of $\mathrm{M}$ cell repolarization [8]. The mechanism is that the potential of $\mathrm{M}$ cells is the highest in the platform phase, the potential difference between $M$ cells and epicardial cells was opposite to that between $M$ cells and endocardial cells, the direction of the potential difference between $M$ cells and epicardial cells was the same as that of the ascending branch of $\mathrm{T}$ wave, but opposite to that of epicardial cells. Therefore, the sum of action potential differences among $M$ cells, epicardial cells and endocardial cells constitutes the ascending branch of $\mathrm{T}$ wave. When the repolarization of epicardial cells ends, the potential difference between $M$ cells and endocardial cells forms the descending branch of T wave. Because the APD of endocardial cells is longer than that of epicardial cells, the potential difference between $M$ cells and endocardial cells limit the height of $\mathrm{T}$ wave peak. Finally, the repolarization of $\mathrm{M}$ cells ended and the $\mathrm{T}$ wave ended accordingly [6]. Therefore, Tp-e and Q-T interval can indirectly reflect TDR. Because Tp-e and Q-T intervals are easily affected by heart rate, this study used $\mathrm{Tp}-\mathrm{ec}, \mathrm{Q}-\mathrm{Tc}$ and $\mathrm{Tp}-\mathrm{e} / \mathrm{Q}-\mathrm{T}$ which were corrected by heart rate to eliminate the interference of heart rate [9]. Because HRV can reflect the activity of cardiac autonomic nervous system and is related to the prognosis and progress of various heart diseases such as coronary heart disease, HRV is included in the research index to obtain a more accurate prediction model.

There was no significant difference in age and gender between the two groups, and the interference of general factors such as age and gender was excluded. There were significant differences in Tp-ec, Q-Tc, Tp-e/Q-T and SDNN between the two groups. The Tp-ec, Q-Tc and Tp-e/Q-T of patients with malignant arrhythmia in hospital were higher than those of normal control group, while 
SDNN was lower than that of normal control group. Multivariate logistic regression was used to obtain the prediction model: $\mathrm{Z}=-254.827+0.203 *$ Tp-ec + $0.581 * \mathrm{Q}-\mathrm{Tc}+878.066 *$ Tp-e/Q-T $-0.248 *$ SDNN. ROC curve was used to test the diagnostic value of each research index and prediction model for the occurrence of malignant arrhythmia in hospital. It was found that the prediction model had the highest diagnostic value, with the area under the curve of 0.993 , followed by Tp-ec, Tp-e/Q-T and Q-Tc, with the area under the curve of 0.988 , $0.973,0.905$ and 0.901 , respectively. $\mathrm{AUC}=0.5$ means that the factor has no diagnostic value, AUC closer to 1 means that the diagnostic value is greater, 0.5 0.7 means that the diagnostic value is lower, $0.7-0.9$ means that the diagnostic value is medium, and 0.9 - 1 means that the diagnostic value is higher. Tp-ec, Q-Tc, Tp-e/Q-T, SDNN and prediction model have high diagnostic value for malignant ventricular arrhythmia in hospital. The best critical value of predictive model for predicting malignant ventricular arrhythmia in hospital was 181.792, its sensitivity and specificity were 0.990 and 0.940 respectively. The optimal cut-off values of Tp-ec, Q-Tc, Tp-e/Q-T and SDNN were $100.365 \mathrm{~ms}, 447.078$ ms, 0.239 and 100.500 respectively. Rautaharju et al. [10] showed that the normal range of $\mathrm{Tp}$-ec was $(85 \pm 11)$ The tp-t/Qt was relatively constant in the range of $0.1-0.25 \mathrm{MS}$, which was consistent with the results of this study.

In conclusion, $\mathrm{Tp}-\mathrm{ec}, \mathrm{Q}-\mathrm{Tc}, \mathrm{Tp}-\mathrm{e} / \mathrm{Q}-\mathrm{T}$ indirectly reflect TDR, HRV reflects cardiac autonomic nerve function, which are related to ventricular malignant arrhythmia, and all have high predictive value. The predictive model which is combined with the above four indicators has the highest predictive value for malignant ventricular arrhythmia in hospital, which is helpful for clinicians to predict early.

\section{Acknowledgements}

The authors thank the patient and his families and all the investigators, including the physicians, nurses, and laboratory technicians in this study.

\section{Conflicts of Interest}

The authors declare no conflicts of interest regarding the publication of this paper.

\section{References}

[1] Mugnai, G., Benfari, G., Fede, A., et al. (2016) Tpeak-to-Tend/QT Is an Independent Predictor of Early Ventricular Arrhythmias and Arrhythmic Death in Anterior ST Elevation Myocardial Infarction Patients. European Heart Journal Acute Cardiovascular Care, 5, 473-480. https://doi.org/10.1177/2048872615598616

[2] Antzelevitch, C. and Di Diego, J.M. (2019) Tpeak-Tend Interval as a Marker of Arrhythmic Risk. Heart Rhythm, 16, 954-955.

https://doi.org/10.1016/j.hrthm.2019.01.017

[3] Fish, J.M., Di Diego, J.M., Nesterenko, V., et al. (2004) Epicardial Activation of Left Ventricular Wall Prolongs QT Interval and Transmural Dispersion of Polarization: Implication for Biventricular Pacing. Criculation, 109, 2136-2142. 
https://doi.org/10.1161/01.CIR.0000127423.75608.A4

[4] Karahan, Z., Ugurlu, M., Ucaman, B., et al. (2016) Association between ACE Gene Polymorphism and QT Dispersion in Patients with Acute Myocardial Infarction. The Open Cardiovascular Medicine Journal, 10, 117-121. https://doi.org/10.2174/1874192401610010117

[5] Besnier, F., Labrunée, M., Richard, L., et al. (2019) Short-Term Effects of a 3-Week Interval Training Program on Heart Rate Variability in Chronic Heart Failure. Annals of Physical and Rehabilitation Medicine, 62, 321-328. https://doi.org/10.1016/j.rehab.2019.06.013

[6] Antzelevitch, C. (2010) M Cells in the Human Heart. Circulation Research, 106, 815-817. https://doi.org/10.1161/CIRCRESAHA.109.216226

[7] Gupta, P., Patel, C., Patel, H., et al. (2008) T (p-e)/QT Ratio as an Index of Arrhythmogenesis. Journal of Electrocardiology, 41, 567-574. https://doi.org/10.1016/j.jelectrocard.2008.07.016

[8] Liu, D.W. and Antzelevith, C. (1995) Clinical Relevance of Cardiac Arrhythmias Generated by after Depolarizations Role of M Cells in the Generation of U Waves, Triggered Activity and Torsade Depointes. Journal of Electrocardiology, 6, 591.

[9] Akboga, M.K., Gulcihan, B.K., Yilmaz, S., et al. (2017) Tp-e Interval and Tp-e/QTc Ratio as Novel Surrogate Markers for Prediction of Ventricular Arrhythmic Events in Hypertrophic Cardiomyopathy. Original Investigation, 18, 48-53.

https://doi.org/10.14744/AnatolJCardiol.2017.7581

[10] Rautaharju, P.M., Zhang, Z.M., Gregg, R.E., et al. (2013) Normal Standards for Computer-ECG Programs for Prognostically and Diagnostically Important ECG Variables Derived from a Large Ethnically Diverse Female Cohort: The Women's Health Initiative (WHI). Journal of Electrocardiology, 46, 707-716. https://doi.org/10.1016/j.jelectrocard.2013.05.136 